\title{
\begin{tabular}{l|l} 
Mitraries & DSpace@MIT
\end{tabular}
}

\author{
MIT Open Access Articles
}

\section{A CR-HYDRO-NEI MODEL OF THE STRUCTURE AND BROADBAND EMISSION FROM TYCHO'S SUPERNOVA REMNANT}

The MIT Faculty has made this article openly available. Please share how this access benefits you. Your story matters.

Citation: Slane, P., S.-H. Lee, D. C. Ellison, D. J. Patnaude, J. P. Hughes, K. A. Eriksen, D. Castro, and S. Nagataki. "A CR-HYDRO-NEI MODEL OF THE STRUCTURE AND BROADBAND EMISSION FROM TYCHO'S SUPERNOVA REMNANT.” The Astrophysical Journal 783, no. 1 (February 10, 2014): 33. (C) 2014 The American Astronomical Society

As Published: http://dx.doi.org/10.1088/0004-637X/783/1/33

Publisher: IOP Publishing

Persistent URL: http://hdl.handle.net/1721.1/93132

Version: Final published version: final published article, as it appeared in a journal, conference proceedings, or other formally published context

Terms of Use: Article is made available in accordance with the publisher's policy and may be subject to US copyright law. Please refer to the publisher's site for terms of use. 


\title{
A CR-HYDRO-NEI MODEL OF THE STRUCTURE AND BROADBAND EMISSION FROM TYCHO'S SUPERNOVA REMNANT
}

\author{
P. Slane ${ }^{1}$, S.-H. LeE ${ }^{2}$, D. C. Ellison ${ }^{3}$, D. J. Patnaude ${ }^{1}$, J. P. Hughes ${ }^{4}$, K. A. Eriksen ${ }^{4,5}$, D. Castro ${ }^{6}$, and S. Nagataki ${ }^{2}$ \\ ${ }^{1}$ Harvard-Smithsonian Center for Astrophysics, 60 Garden Street, Cambridge, MA 02138, USA; slane@ cfa.harvard.edu, dpatnaude@ cfa.harvard.edu \\ ${ }^{2}$ RIKEN, Astrophysical Big Bang Laboratory, 2-1 Hirosawa, Wako, Saitama 351-0198, Japan; shiu-hang.lee@ riken.jp, shigehiro.nagataki@ riken.jp \\ ${ }^{3}$ Physics Department, North Carolina State University, Box 8202, Raleigh, NC 27695, USA; don_ellison@ncsu.edu \\ ${ }^{4}$ Department of Physics and Astronomy, Rutgers University, Piscataway, NJ 08854-8019, USA; jph@physics.rutgers.edu \\ ${ }^{5}$ XTD-IDA, Los Alamos National Laboratory, P.O. Box 1663, Los Alamos, NM 87545, USA; keriksen@lanl.gov \\ ${ }^{6}$ MIT-Kavli Center for Astrophysics and Space Research, 77 Massachusetts Avenue, Cambridge, MA 02139, USA; castro@mit.edu \\ Received 2013 October 17; accepted 2014 January 10; published 2014 February 10
}

\begin{abstract}
Tycho's supernova remnant (SNR) is well-established as a source of particle acceleration to very high energies. Constraints from numerous studies indicate that the observed $\gamma$-ray emission results primarily from hadronic processes, providing direct evidence of highly relativistic ions that have been accelerated by the SNR. Here we present an investigation of the dynamical and spectral evolution of Tycho's SNR by carrying out hydrodynamical simulations that include diffusive shock acceleration of particles in the amplified magnetic field at the forward shock of the SNR. Our simulations provide a consistent view of the shock positions, the nonthermal emission, the thermal X-ray emission from the forward shock, and the brightness profiles of the radio and X-ray emission. We compare these with the observed properties of Tycho to determine the density of the ambient material, the particle acceleration efficiency and maximum energy, the accelerated electron-to-proton ratio, and the properties of the shocked gas downstream of the expanding SNR shell. We find that evolution of a typical Type Ia supernova in a low ambient density $\left(n_{0} \sim 0.3 \mathrm{~cm}^{-3}\right)$, with an upstream magnetic field of $\sim 5 \mu \mathrm{G}$, and with $\sim 16 \%$ of the SNR kinetic energy being converted into relativistic electrons and ions through diffusive shock acceleration, reproduces the observed properties of Tycho. Under such a scenario, the bulk of observed $\gamma$-ray emission at high energies is produced by $\pi^{0}$-decay resulting from the collisions of energetic hadrons, while inverse-Compton emission is significant at lower energies, comprising roughly half of the flux between 1 and $10 \mathrm{GeV}$.
\end{abstract}

Key words: acceleration of particles - cosmic rays - ISM: individual objects (Tycho's SNR) - ISM: supernova remnants - shock waves

Online-only material: color figures

\section{INTRODUCTION}

Efficient acceleration of charged particles in the shocks of supernova remnants (SNRs) has long been cited as a likely process through which a significant fraction of Galactic cosmicrays (CRs) are accelerated. The evidence for such energetic particles is compelling. Radio emission from SNRs originates from electrons with energies $E_{e}>1 \mathrm{GeV}$, while observations of nonthermal X-ray emission from SNRs reveal electrons with energies exceeding tens of TeV. Evidence for energetic ions accelerated in SNRs is more elusive because of the low radiation efficiency for such particles. The observed $\mathrm{GeV}$ and $\mathrm{TeV} \gamma$-ray emission from many SNRs-particularly those known to be located in dense environments - is consistent with the presence of energetic protons that produce $\gamma$-rays through the decay of neutral pions created in collisions with ambient nuclei. But $\gamma$-rays can also be produced from the energetic electron population, through inverse-Compton (IC) scattering of ambient photons or through nonthermal bremsstrahlung. Modeling of the broadband spectra from such SNRs, in order to ascertain the nature of the $\gamma$-ray emission, is complicated and has led to mixed interpretations, making the evidence for ion acceleration controversial in some cases. Gamma-ray emission from some SNRs known to be interacting with molecular clouds seems to require a significant component from pion decay (e.g., Abdo et al. 2009, 2010; Castro \& Slane 2010; Giuliani et al. 2011; Ackermann et al. 2013), with some remnants requiring nearly all of the SNR kinetic energy to be converted to relativistic electrons for IC emission to dominate the flux (Castro et al. 2013; Auchettl et al. 2014). For W44 and IC 443, the $\gamma$-ray spectra show clear evidence of a kinematic "pion bump," firmly establishing the presence of energetic ions in these remnants (Ackermann et al. 2013).

Ion acceleration can have observable dynamical effects on SNR evolution, since this process results in less thermal heating of the swept-up gas and an increased compression ratio in the postshock region. X-ray studies of 1E0102 (Hughes et al. 2000) reveal electron temperatures that are much lower than expected from the observed expansion velocities of the remnants, for example, indicating that a large fraction of the shock energy has gone into something other than thermal heating of the gas. In addition, as we discuss in more detail below, X-ray studies of Tycho's SNR (Warren et al. 2005) demonstrate that the ratio of the forward shock (FS) radius to that of the contact discontinuity (CD), as well as that of the reverse shock (RS), is smaller than expected, consistent with results expected from efficient ion acceleration (Decourchelle et al. 2000; Ellison et al. 2004). Finally, deep Chandra observations of Tycho reveal a complex of regularly spaced stripe-like nonthermal structures in the projected interior of the remnant (Eriksen et al. 2011). The spacing of these stripes may correspond to the gyroradii of $10^{14}-10^{15} \mathrm{eV}$ protons in an amplified magnetic field (Eriksen et al. 2011), although Bykov et al. (2011) suggest that the structures may be the result of anisotropic magnetic turbulence 
produced by instabilities driven by $\mathrm{CR}$ protons with energies of $\sim 10^{15} \mathrm{eV}$. The maximum energy to which such ions are accelerated in a particular SNR is of critical importance to our understanding of the role SNRs play in producing ions with energies approaching the knee of the CR spectrum. Combined with modeling of the broadband emission that appears to imply that pion decay dominates the $\gamma$-ray flux (Morlino \& Caprioli 2012; Giordano et al. 2012; Berezhko et al. 2013; Zhang et al. 2013), these observations have thus led to the conclusion that Tycho's SNR is a particularly important testbed for models of particle acceleration in SNRs.

The thermal emission from material compressed by the FS provides particularly important information on particle acceleration in SNRs. Because the postshock temperature is reduced in the case of efficient acceleration, the emission characteristics of the plasma are modified. Moreover, the ionization state of the gas is modified by both the reduced temperature and the higher density associated with the increased compression ratio (Patnaude et al. 2009). As a result, self-consistent treatment of the thermal emission is crucial in any effort to model the effects of CR acceleration in SNRs. This is of particular importance in assessing the nature of any observed $\gamma$-ray emission because of the critical dependence on density shared by both $\pi^{0}$-decay emission and thermal X-ray emission. For example, the lack of observed thermal X-ray emission from RX J1713.7-3946 eliminates $\pi^{0}$-decay as a significant contributor to the $\gamma$-ray emission (Ellison et al. 2010, 2012) unless the postshock medium is filled with cold, clumped gas (Inoue et al. 2012). Conversely, the density required to produce the observed thermal X-ray emission from H-like ions of $\mathrm{Si}$ in CTB 109 is sufficiently high for $\pi^{0}$-decay to account for roughly half of the observed $\gamma$-ray flux (Castro et al. 2012). Any complete picture of the CR modified emission and dynamical evolution of Tycho's SNR must include a self-consistent treatment of thermal X-ray emission.

Tycho's SNR is the product of SN 1572. Based on historical records of its light curve, the remnant has long been understood to have resulted from a Type Ia event (Ruiz-Lapuente 2004), corresponding to the thermonuclear destruction of a $\mathrm{C}-\mathrm{O}$ white dwarf star. This has been confirmed through direct measurements of the supernova spectrum, taken from light echo measurements (Krause et al. 2008) that identify it as belonging to the normal Type Ia class of SNe. As with many Galactic SNRs, the distance to Tycho's SNR is rather uncertain. Most estimates fall in the $2-5 \mathrm{kpc}$ range with recent estimates of $4 \pm 1 \mathrm{kpc}$ based on observed ejecta velocities and proper motion measurements (Hayato et al. 2010), 3.8 $8_{-0.9}^{+1.5} \mathrm{kpc}$ based on light echo measurements (Krause et al. 2008), and 2.5-3 kpc based on kinematic methods (Tian \& Leahy 2011). Morlino \& Caprioli (2012) estimate $d=3.3 \mathrm{kpc}$ based on broadband modeling of the spectrum (see Section 4 below), although their analysis is based on the Truelove \& McKee (1999) parameterization of the SNR evolution rather than a self-consistent hydrodynamical treatment that includes the effects of the CR acceleration, such as that used here.

The X-ray emission from Tycho's SNR (Figure 1) is dominated by ejecta (Hwang \& Gotthelf 1997; Decourchelle et al. 2001; Hwang et al. 2002), accompanied by thin filaments of synchrotron emission from extremely energetic electrons accelerated at the FS (Cassam-Chenaï et al. 2004; Eriksen et al. 2011; Bykov et al. 2011). The mean angular radius of the remnant is $251^{\prime \prime}$, with an azimuthal variation of about $\pm 16^{\prime \prime}$ (Warren et al. 2005). Comparison of the ejecta density and composition structure with hydrodynamical models for the evolution and nucleosynthesis models for the ejecta produced in different classes of Type Ia explosions indicate that the remnant is the result of a delayed-detonation explosion (Badenes et al. 2006) with a $\sim 10^{51} \mathrm{erg}$ explosion expanding into an ambient density $n_{0}=0.85-2.1 \mathrm{~cm}^{-3}$, although most other studies indicate lower densities: $n_{0} \lesssim 0.3 \mathrm{~cm}^{-3}$ based on limits to the thermal X-ray emission (Cassam-Chenaï et al. 2007); $n_{0} \lesssim 0.4 \mathrm{~cm}^{-3}$ based on the $\gamma$-ray flux (Völk et al. 2008); $n_{0} \lesssim 0.2 \mathrm{~cm}^{-3}$ based on measurements of the SNR expansion index $m$, where $R \propto t^{m}$ (Katsuda et al. 2010); and $n_{0} \sim 0.1-0.2 \mathrm{~cm}^{-3}$ (except in distinct regions of known dense clump interactions) based on the ratio of 70 to $24 \mu \mathrm{m}$ flux ratios from postshock dust in Tycho (Williams et al. 2013).

Gamma-ray emission from Tycho has been identified by observations with VERITAS (Acciari et al. 2011) and the Fermi Large Area Telescope (LAT; Giordano et al. 2012), and models of the broadband spectrum indicate that the $\gamma$-ray flux is dominated by emission from $\pi^{0}$-decay (Morlino \& Caprioli 2012; Berezhko et al. 2013; Zhang et al. 2013), although the details of the models used to reach this conclusion differ considerably. In addition, arguments have been made for models in which the $\gamma$-rays may be dominated by IC emission (Atoyan \& Dermer 2012).

Here we present a study of the radial structure, evolution, and broadband emission from Tycho's SNR using hydrodynamical simulations described in Section 2. In Section 3 we describe our modeling approach and summarize the application of these models to the broadband spectral energy distribution (SED) for Tycho as well as the X-ray emission from the postshock region of the SNR blast wave. We discuss the results of our modeling efforts in Section 4, in the context of previous studies of Tycho, and our conclusions are presented in Section 5. We confirm, in what we believe to be the most complete, spherically symmetric, broadband model of this SNR yet performed, that the bulk of the $\gamma$-ray emission is from hadronic processes with a significant fraction of $\gamma$-ray emission contributed by leptonic processes at $\mathrm{GeV}$ energies. Other important properties of the SNR, such as ambient density, magnetic field strength, the diffusive shock acceleration (DSA) efficiency, and the relativistic electron-toproton ratio, are also constrained.

\section{THE CR-HYDRO-NEI MODEL}

To address the evolution, particle acceleration, and broadband emission for Tycho's SNR, we have used the CR-hydro-NEI code that models the SNR hydrodynamics with a version of the VH-1 hydro code (e.g., Blondin \& Ellison 2001) modified to include the effects of nonlinear DSA using a semi-analytic solution based on the treatments from Blasi et al. (2005) and Caprioli et al. (2009). The resulting nonthermal proton and electron spectra, coupled with the calculated (amplified) magnetic field and assumed ambient photon fields, are used to calculate the synchrotron, bremsstrahlung, IC, and $\pi^{0}$-decay emission. The thermal X-ray emission is calculated by following the ionization of the shocked gas through the hydro simulation and coupling this to a non-equilibrium ionization emission code (e.g., Ellison et al. 2007; Patnaude et al. 2009). A full description of the CR-hydro-NEI code can be found in Lee et al. (2012). The approach here is similar to that used for investigations of RX J1713.7-3946 (Ellison et al. 2012), CTB 109 (Castro et al. 2012), and Vela Jr. (Lee et al. 2013).

We note that while our model is spherically symmetric, it is inhomogeneous in radius. We begin with a radial ejecta density 


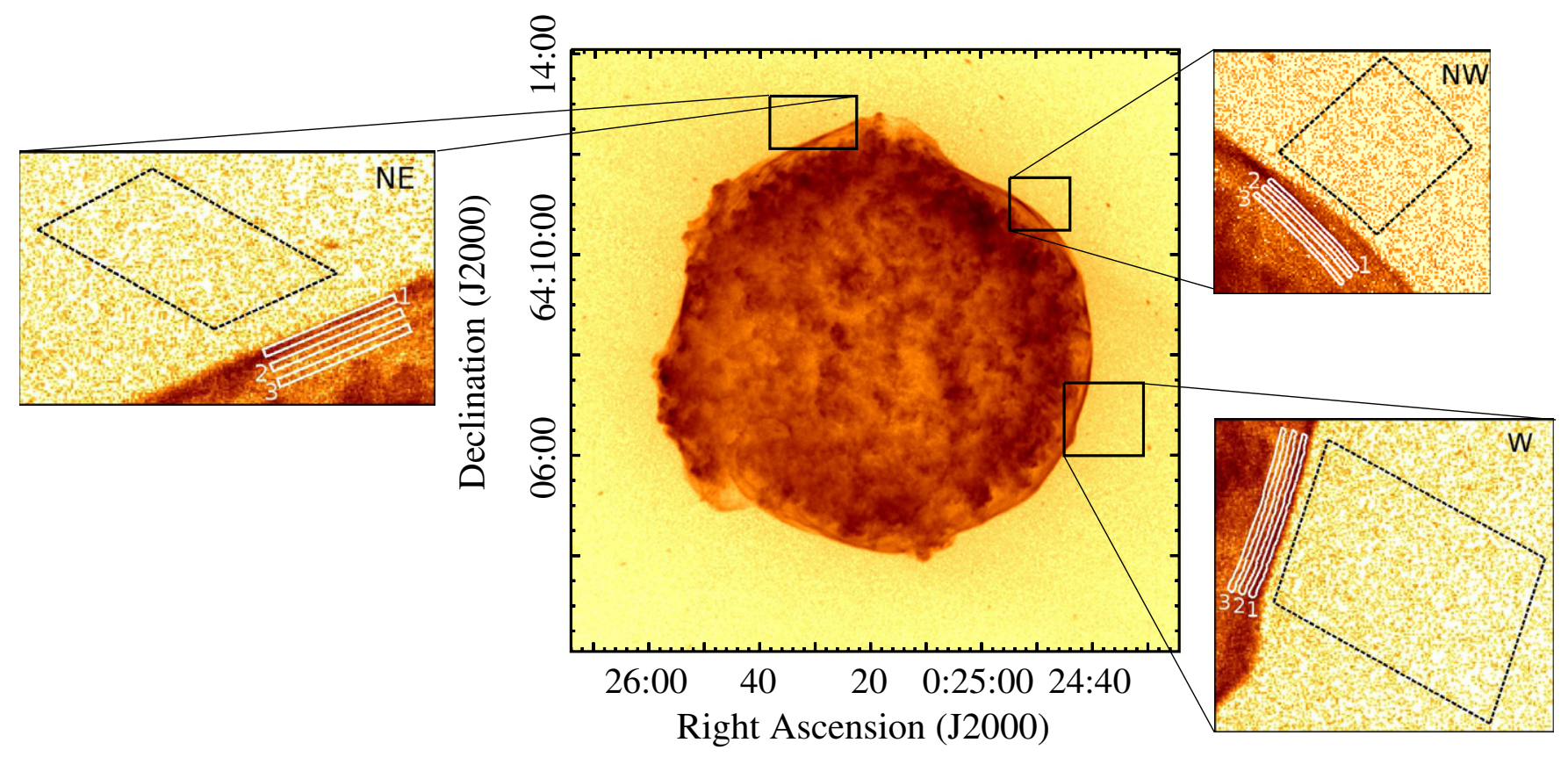

Figure 1. Chandra image of Tycho's SNR. Insets identify regions used for spectral extraction, with dashed regions indicating background.

(A color version of this figure is available in the online journal.)

distribution (assumed here to be exponential, with a total mass of $\left.1.4 M_{\odot}\right)$, and all parameters including the density, temperature, magnetic field, and ionization state, evolve as the remnant ages and shocked material advects downstream from the FS. At the current age, the relativistic electrons and ions, as well as the thermal plasma, emit from a "continuous zone" environment between the CD and FS. Rather then assuming a single power law with an exponential cutoff for the acceleration CRs, as in some models (e.g., Zhang et al. 2013), with the power-law index and cutoff parameter fixed to match the data, we determine the evolving full particle spectrum, spatially resolved and integrated over time, using a self-consistent model.

\section{MODELING}

The primary parameters for driving the simulations are the SNR distance $(d)$, the upstream (i.e., unshocked) density $\left(n_{0}\right)$ and the upstream magnetic field $\left(B_{0}\right.$; both assumed constant here), the kinetic energy of the explosion $\left(E_{51}\right.$, in units of $\left.10^{51} \mathrm{erg}\right)$, and the DSA injection efficiency parameter $(\xi)$. We investigated a range of distances from $2.5-5 \mathrm{kpc}$, and explored a grid of values for the other key parameters for each assumed distance until the observed angular radius was reproduced at the known age of Tycho. We compared the angular positions of the RS and $\mathrm{CD}$ for each model with the measured values from Warren et al. (2005), and the predicted angular expansion speed with measurements from Katsuda et al. (2010), and rejected models for which the discrepancy was larger than $\sim 0.5$ arcmin. We initially assumed expansion into an interstellar medium (ISM) with constant density $n_{0} \lesssim 0.3 \mathrm{~cm}^{-3}$, based on upper limits established by Katsuda et al. (2010), and adopted $E_{51}=1$ based on the spectral classification of Tycho's SN as a normal Type Ia event (Krause et al. 2008).

For models that satisfied the above conditions, we varied additional parameters that primarily impact the spectrum in subsequent CR-hydro-NEI runs. These include the electron-toproton number density ratio, $K_{\mathrm{ep}}$, a shape parameter for spectral cutoff around the maximum momentum, $\alpha_{\text {cut }}$ (see Lee et al. 2012), the ratio of the wave damping and growth rates in the acceleration region, and the spatial variation of the Alfvén speed in the shock precursor, $f_{\text {alf }}$.

Using the shocked downstream and precursor magnetic field and plasma density provided by the hydro calculations, we calculated the synchrotron, IC, and nonthermal bremsstrahlung emission from the relativistic electrons. For the IC emission, we used seed photon fields from the cosmic microwave background (CMB), starlight, and IR emission from local dust, to which we also added a local IR field from Tycho itself based on Akari observations (Ishihara et al. 2010). Emission from $\pi^{0}$-decay was calculated based on the model from Kamae et al. (2006). Synchrotron, IC, and nonthermal bremsstrahlung emission from secondary electrons was calculated as well, though these did not contribute significantly to the overall emission.

For models that adequately reproduced the broadband SED for Tycho, we used the predicted X-ray emission models as templates for fitting Chandra spectra taken from regions along and just behind the FS of the SNR (see Figure 1).

\subsection{Parameter Studies}

To arrive at a self-consistent model for Tycho's SNR, we first explored the global parameter space by investigating the effects of varying $d, n_{0}, B_{0}$, and $\xi$. These results are summarized in Figure 2 where we plot the variation with ambient density $n_{0}$ of the angular radius of Tycho, its angular expansion rate, and the value of $\log E^{2} d N / d E$ at fiducial energy values characterizing the radio, nonthermal X-ray, high-energy and very-high-energy $\gamma$-ray bands. ${ }^{7}$ For each panel, horizontal bands indicate the observed uncertainty range for the identified quantity, and the different curves connect model calculations for different values of the efficiency, distance, and unshocked magnetic field, as indicated.

\footnotetext{
7 While Figure 2 displays results only for $B=5 \mu \mathrm{G}$, models with values between 3 and $20 \mu \mathrm{G}$ were investigated.
} 

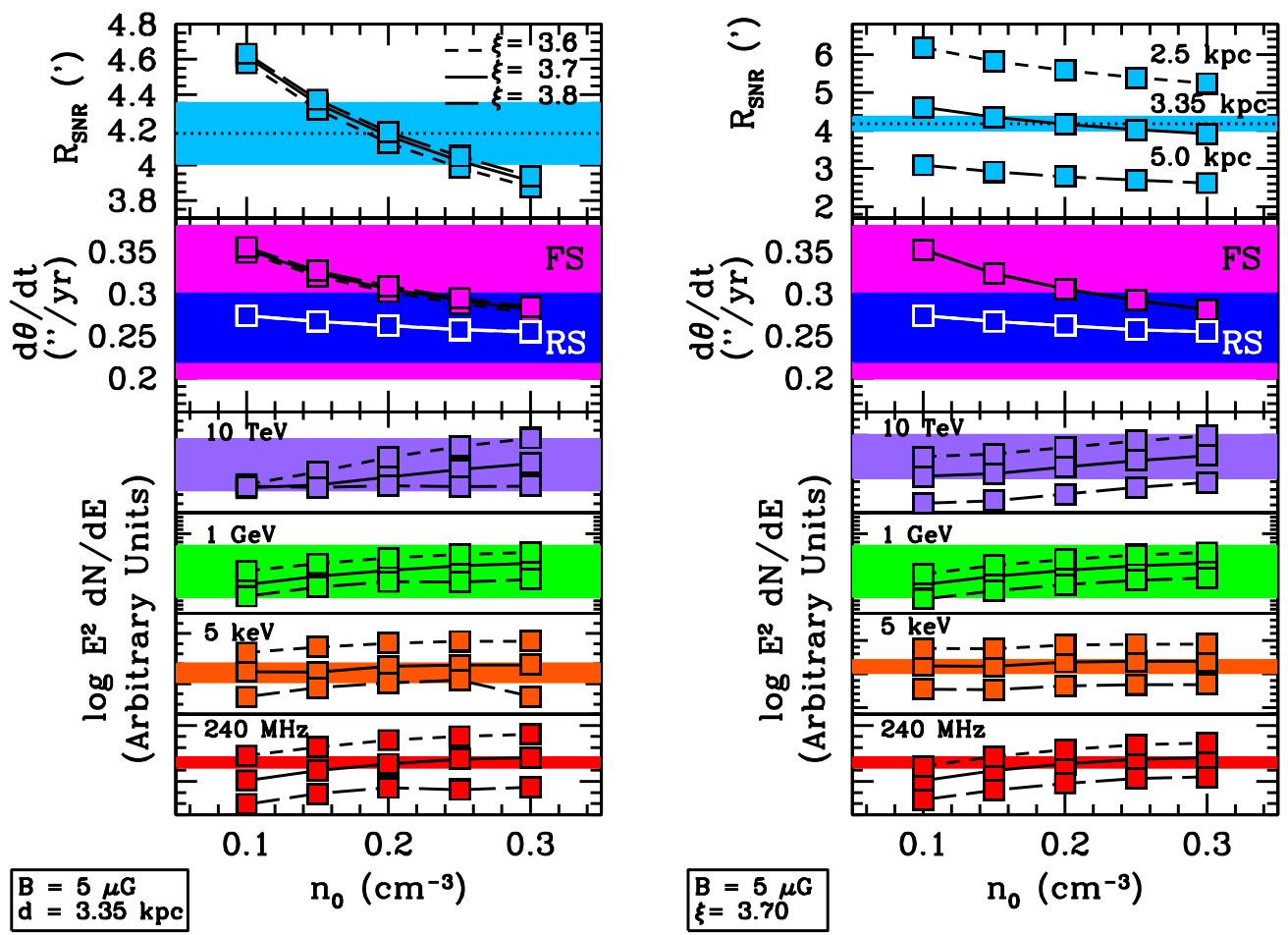

Figure 2. Comparison of model predictions for the angular size, $R_{\mathrm{SNR}}$, the angular expansion rate, $d \theta / d t$, for the FS and RS, and the radio, X-ray, and $\gamma$-ray fluxes for Tycho's SNR as a function of ambient density. The left panel shows model results for different DSA injection parameters, $\xi$, for fixed values for the distance and upstream magnetic field. Note that smaller values of $\xi$ imply larger acceleration efficiencies (see Table 1). The right panel shows results for different distance values for fixed efficiency and magnetic field values.

(A color version of this figure is available in the online journal.)

Table 1

CR-hydro-NEI Model Parameters

\begin{tabular}{llll}
\hline \hline Parameter & Model A & Model B & Model C \\
\hline \multicolumn{4}{c}{ Input } \\
\hline$d(\mathrm{kpc})$ & 3.18 & 3.40 & 2.90 \\
$n_{0}\left(\mathrm{~cm}^{-3}\right)$ & 0.3 & 0.2 & 0.85 \\
$f_{\text {alf }}$ & 0.7 & 0.5 & 0.5 \\
$\xi$ & 3.6 & 3.7 & 3.9 \\
$K_{\text {ep }}$ & 0.003 & 0.008 & 0.01 \\
\hline \multicolumn{4}{c}{ Output } \\
$M_{\text {sw }}\left(M_{\odot}\right)$ & 2.47 & 2.02 & 5.33 \\
$R_{\text {tot }}$ & 4.64 & 4.48 & 4.23 \\
$E_{\mathrm{CR}} / E_{\mathrm{SN}}$ & 0.16 & 0.11 & 0.07 \\
Eff $_{\mathrm{DSA}}$ & 0.26 & 0.20 & 0.11 \\
$p_{\text {max }} / 10^{4} m_{p} c$ & 4.3 & 9.5 & 5.8 \\
\hline
\end{tabular}

Note. $E_{51}=1, B=5 \mu \mathrm{G}$, and $\alpha_{\text {cut }}=0.5$ for all models.

The broad parameter space investigation above produced reasonable models for input values of $d \sim 3 \mathrm{kpc}, n_{0} \sim 0.2 \mathrm{~cm}^{-3}$, $B_{0} \sim 5 \mu \mathrm{G}$, and $\xi \sim 3.7$. We then refined these parameters along with $K_{e-p}$, and $f_{\text {alf }}$ until the predicted broadband spectrum provided a good agreement with the observations. The upper panel in Figure 3 shows the results for our best model (Model A), whose parameters are summarized in Table 1 . We plot the radial variation of the density in order to best illustrate the model positions for the FS, CD, and RS. The observed average FS radius is indicated in green, and the uncertainty range for the $\mathrm{CD}(\mathrm{RS})$ is indicated in magenta (red). While there is actually an observed variation of about $6 \%$ in the FS radius for Tycho, all models were constructed to yield the same outer shock position, to facilitate more direct comparison.
The dashed curve in the upper panel of Figure 3 corresponds to the same input parameters as for Model A except that efficient particle acceleration has been turned off. As expected, the FS/RS and FS/CD radius ratios are smaller for Model A as a result of particle acceleration. The RS position for Model A, which we note is sensitive to the assumed density profile of the ejecta, is in good agreement with that observed by Warren et al. (2005). The radius of the CD falls considerably short of that inferred from the data. This may be the result of Rayleigh-Taylor (R-T) instabilities at the CD resulting in the penetration of ejecta into the shocked ISM. Such instabilities in SN Ia remnants were studied by Wang \& Chevalier (2001), who found that these structures can extend as much as $\sim 11 \%$ beyond the $\mathrm{CD}$ radius at the dynamical age of Tycho's SNR. This effective $\mathrm{R}-\mathrm{T}$ extension region is indicated in cyan in the upper panel of Figure 3. Since the CD region identified by Warren et al. (2005) corresponds to the interface between ejecta emission and the shocked ISM, it is clear that ejecta-filled filaments extending from the CD in Model A can explain the inferred position of this interface. In addition, simulations show that, in the case of efficient particle acceleration, such R-T structures can extend to even larger distances (Blondin \& Ellison 2001; Warren \& Blondin 2013). Thus, we contend that the positions of the RS, FS, and CD in Model A are in good agreement with observations.

\subsection{Broadband SED}

The lower panel in Figure 3 presents the predicted spectrum for Model A, along with the observed spectral measurements for Tycho. Radio points were taken from Reynolds \& Ellison (1992), and the nonthermal X-ray spectrum (5-10 keV) shown in blue is from Suzaku observations based on $444 \mathrm{ks}$ of exposure (ObsIDs: 500024010, 503085010, and 503085020), including 

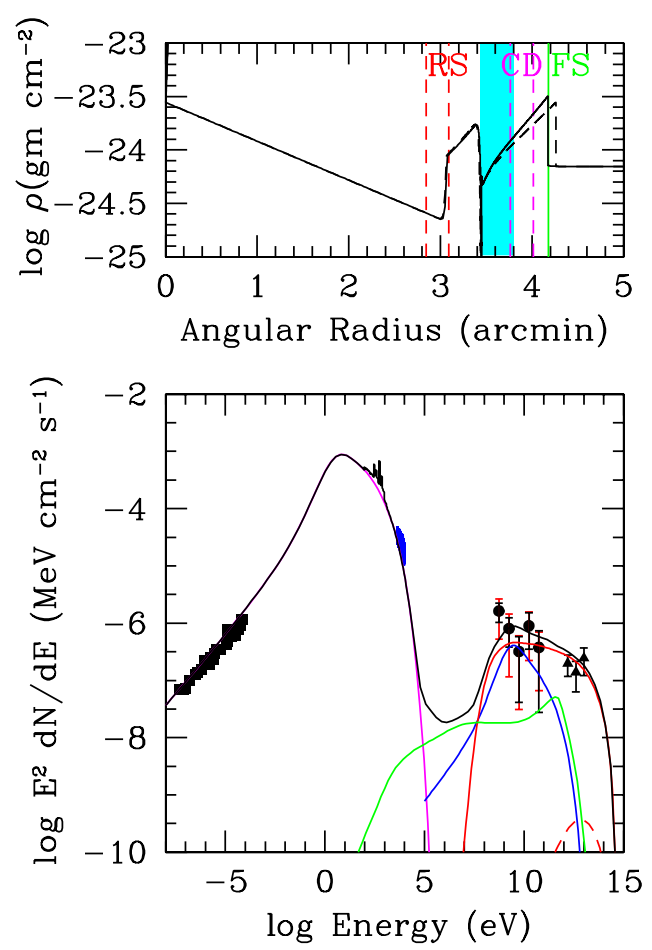

Figure 3. Top: radial density profile for Model A. The black dashed curve corresponds to the same parameters as Model A, but with the particle acceleration effectively turned off. The green vertical line indicates the position of the FS, while the magenta and red lines delineate the $\mathrm{CD}$ and RS ranges reported by Warren et al. (2005). The cyan band indicates the extent of expected R-T mixing based on the work of Wang \& Chevalier 2001). Bottom: broadband SED for Model A, compared with observed emission. See text for description of different curves.

(A color version of this figure is available in the online journal.)

$350 \mathrm{ks}$ from the Tycho key project (PI: Hughes). We use a local background from the outskirts of the field of view and consider spectra only from the front-side illuminated chips (XIS0 and XIS3), which we combined into a single, merged spectrum and appropriate response file. The best fit over the 5-10 keV band (using a model with a single power law and five Gaussians to describe the thermal lines) yields a power-law photon index of $2.83 \pm 0.01$ with a normalization of 0.116 photons $\mathrm{cm}^{-2} \mathrm{~s}^{-1} \mathrm{keV}^{-1}$, values that are consistent with previous measurements. For Figure 3 we plot only the best-fit power-law component with estimated error bars. Black circles represent data from the Fermi-LAT (Giordano et al. 2012) while triangles correspond to data from VERITAS observations (Acciari et al. 2011). For the models, the magenta curve represents the synchrotron emission, the blue curve is from IC emission, the red curve is from $\pi^{0}$-decay, and the green curve is the nonthermal bremsstrahlung emission. The dashed red curve represents $\pi^{0}$-decay emission from escaping protons, and the black curve is the sum of these model components. Weak thermal line features in the soft X-ray band $(\sim 0.2-2.5 \mathrm{keV})$, from the shocked ISM can be seen in the model. (We note that interstellar absorption has not been applied to the SED.)

The results of this model indicate that the $\gamma$-ray emission from Tycho is dominated by $\pi^{0}$-decay at high energies, although the IC emission at lower energies is very significant as well, and makes a nearly equal contribution between 1 and $10 \mathrm{GeV}$. This conclusion is consistent with those presented in several recent studies of Tycho, although our modeling approach contains

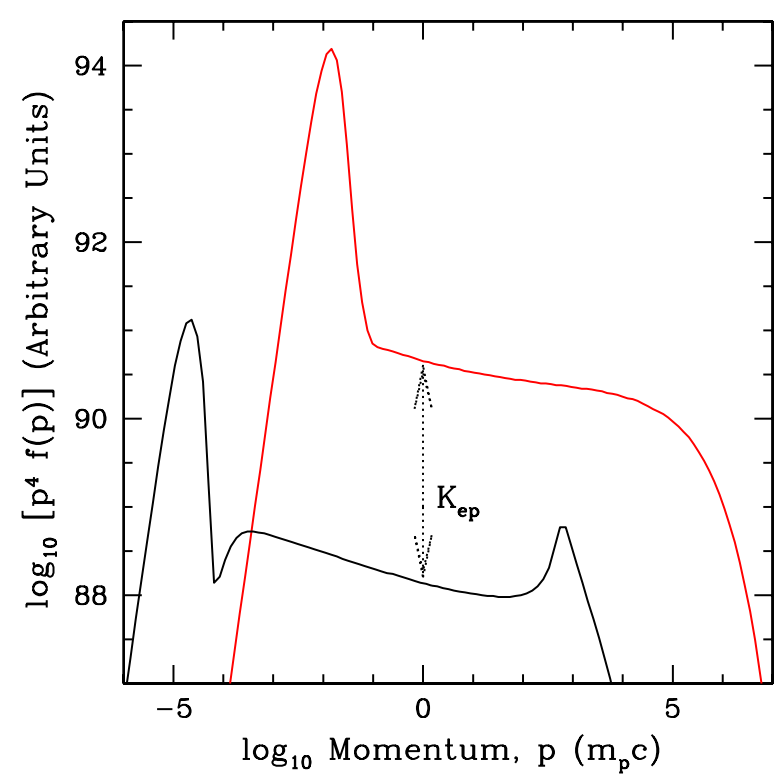

Figure 4. Proton (red) and electron (black) spectra $p^{4} f(p)$ for Model A. (A color version of this figure is available in the online journal.)

important differences, as we discuss below. We note that that the radio spectrum presented in Figure 3 corresponds to the entire SNR while our modeled emission corresponds to the FS. While significant particle acceleration is not expected at the RS, any such component would not be accounted for in the model.

The results of the evolution for Tycho predicted by Model A are summarized in Table 1. At its current age, the remnant has swept up $\sim 2.5 M_{\odot}$ of ISM material. The remnant has placed roughly $16 \%$ of the SNR kinetic energy into relativistic particles, with a total DSA acceleration efficiency of $\sim 26 \%$. The total compression ratio from the FS, $R_{\text {tot }}$, is about $16 \%$ higher than expected for the case of no particle acceleration. The electron and proton momentum spectra for Model A are shown in Figure 4; the maximum proton energy is nearly $50 \mathrm{TeV}$.

Figure 5 shows the results from our Model B (see Table 1), for which the density is slightly lower than that of Model A. The dynamical results are in agreement with the measurements of Warren et al. (2005), although the RS position is just barely consistent with the measured value. We note that recent Suzaku measurements suggest that the RS radius may be even smaller than previously recognized (Yamaguchi et al. 2014), potentially indicating that the position in this model (and perhaps that in Model A) is too large. Model B represents our best model in which the $\gamma$-ray emission is dominated by IC emission, although the highest energies are still dominated by the $\pi^{0}$-decay component. However, the overall fit to both the $\mathrm{GeV}$ and $\mathrm{TeV}$ data is rather poor indicating that a model where leptons dominate the $\gamma$-ray flux is unlikely for Tycho.

In Figure 6 we present the time evolution of the FS (black) and RS (red) angular speeds for our models. Horizontal lines indicate the range of measured values for the angular expansion rate of the FS and of the inner edge of the shocked ejecta which, particularly for the FS, show considerable azimuthal variations (Katsuda et al. 2010). Both models A and B adequately reproduce the observed expansion rates.

\subsection{X-Ray Emission}

The X-ray image in Figure 1 was created by merging observations from a $750 \mathrm{ks}$ Chandra observation carried out 

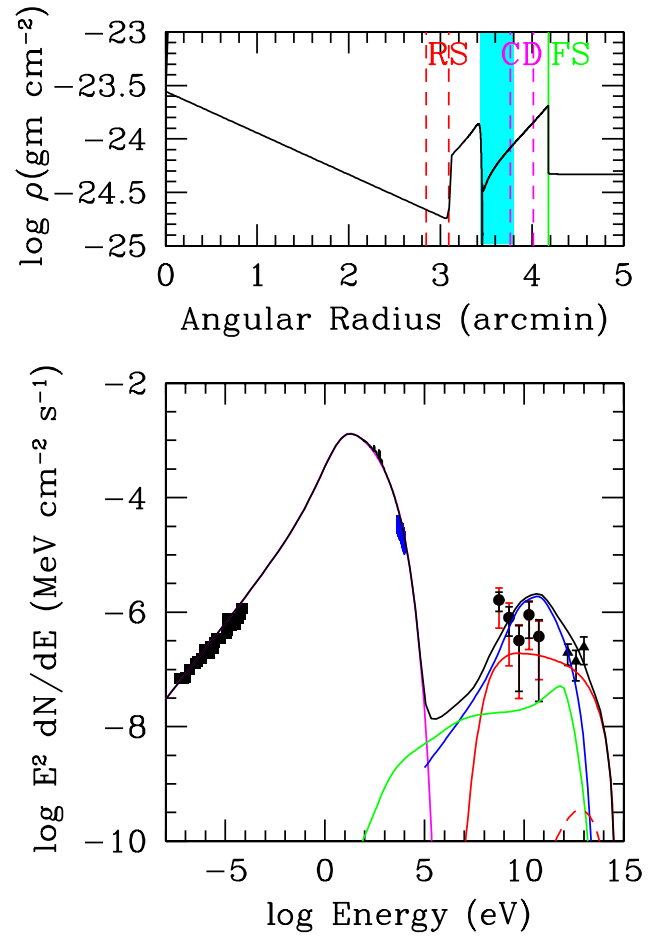

Figure 5. Same as Figure 5, for Model B.

(A color version of this figure is available in the online journal.)

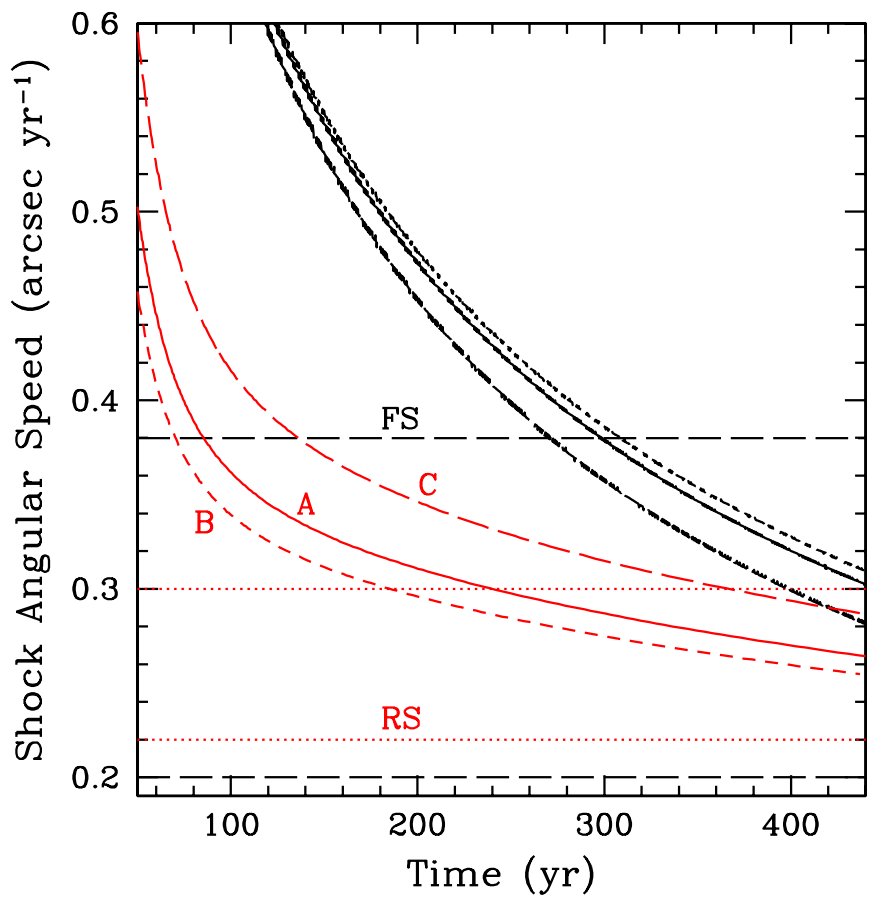

Figure 6. Time evolution of the FS and RS angular speeds for models described in the text. Horizontal dashed curves outline the measured values for the FS (black) and RS (red). Solid lines correspond to Model A while short (long) dashed lines correspond to Model B (C).

(A color version of this figure is available in the online journal.)

in 2009 (ObsIDs 10093-10097, 10902-10904, 10906). Standard cleaning procedures were used on each individual data set, and the resulting images were merged, resulting in a net exposure of $734.1 \mathrm{ks}$. Spectra were extracted from each of the rectangular regions in the northeastern, northwestern, and western portions of the SNR indicated in Figure 1 (hereafter referred to as NE,

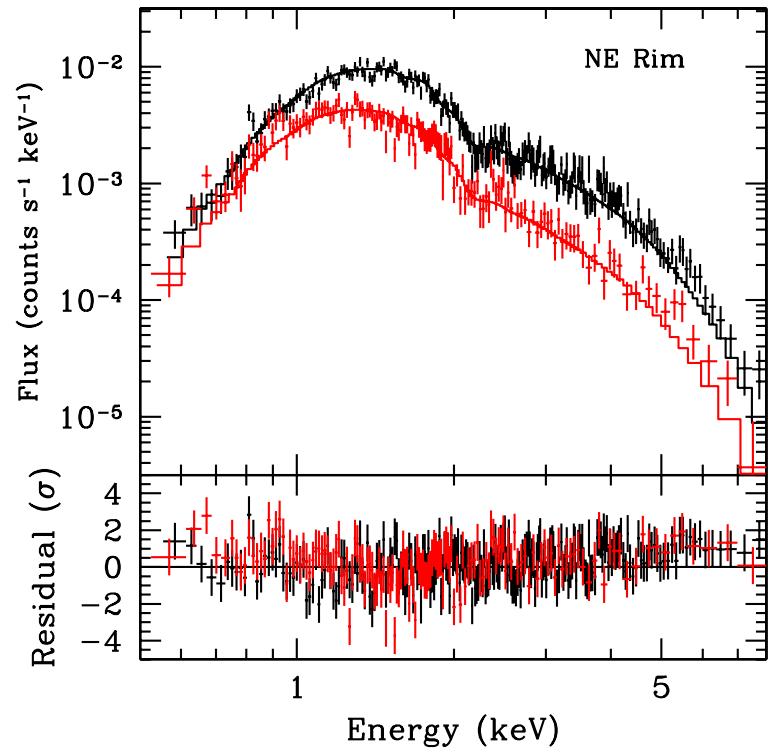

Figure 7. Chandra spectra from the NE rim of Tycho, compared with the predictions from Model A. The upper (black) spectrum corresponds to a region directly along the shock (region 1 in Figure 1) while the lower (red) spectrum is taken from a region immediately behind the shock (region 2).

(A color version of this figure is available in the online journal.)

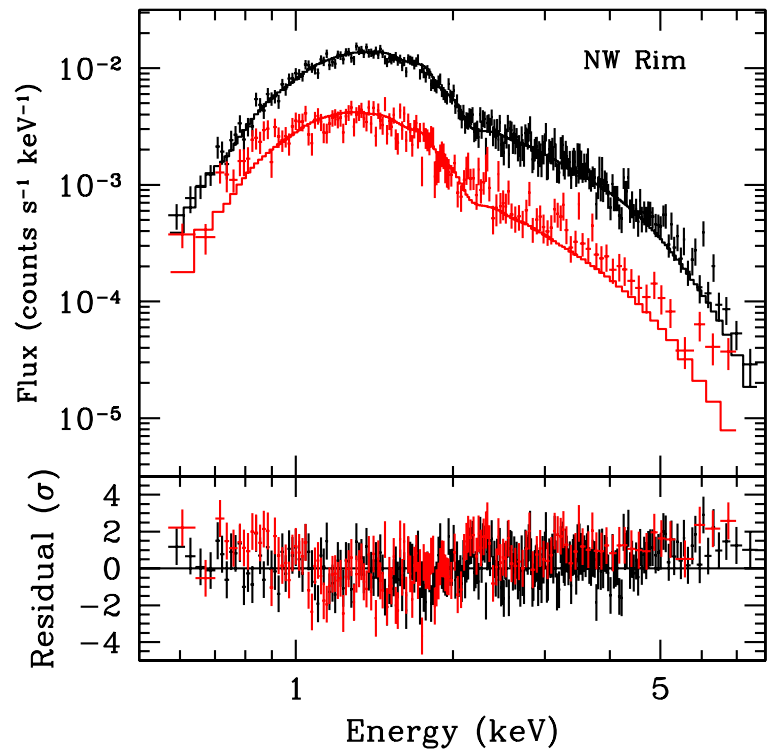

Figure 8. Same as Figure 8, for NW rim of Tycho.

(A color version of this figure is available in the online journal.)

NW, and W regions). These regions were selected because the nonthermal emission is well separated from the bright ejecta; they correspond closely with those used by Cassam-Chenaï et al. (2007). The large outer boxes in each region were used for extraction of background spectra. For each region, spectra from individual ObsIDs were combined using the ciao task dmtcalc, and weighted arf (effective area) and rmf (spectral redistribution) files were created using the addresp task.

The X-ray spectra from directly along the FS and from the region immediately behind the shock in the NE, NW, and $\mathrm{W}$ regions of the SNR are shown in Figures 7-9. Fits were performed with xspec version 12.7.1 using the thermal and nonthermal X-ray emission models calculated in the CR-hydro-NEI runs along with the interstellar absorption model 
Table 2

X-Ray Spectral Fits for Models A, B, and C

\begin{tabular}{|c|c|c|c|c|c|c|c|c|c|}
\hline \multirow[t]{2}{*}{ Parameter } & \multicolumn{3}{|c|}{$\mathrm{NW}^{\mathrm{a}}$} & \multicolumn{3}{|c|}{$\mathrm{NE}^{\mathrm{a}}$} & \multicolumn{3}{|c|}{$\mathrm{W}^{\mathrm{a}}$} \\
\hline & A & $\mathrm{B}$ & $\mathrm{C}$ & A & $\mathrm{B}$ & $\mathrm{C}$ & A & $\mathrm{B}$ & $\mathrm{C}$ \\
\hline$N_{\mathrm{H}}^{\mathrm{b}}$ & $6.3 \pm 0.02$ & $5.3 \pm 0.02$ & $8.9 \pm 0.01$ & $6.6 \pm 0.02$ & $5.7 \pm 0.02$ & $8.7 \pm 0.02$ & $8.0 \pm 0.02$ & $6.9 \pm 0.02$ & $8.9 \pm 0.01$ \\
\hline$K_{1}^{\mathrm{c}}$ & $71.8 \pm 1.6$ & $35.5 \pm 0.8$ & $80.8 \pm 1.7$ & $23.3 \pm 0.6$ & $11.4 \pm 0.4$ & $26.0 \pm 0.7$ & $101.0 \pm 1.3$ & $49.7 \pm 0.9$ & $110.0 \pm 1.7$ \\
\hline$K_{2}^{c}$ & $18.4 \pm 0.6$ & $6.5 \pm 0.2$ & $13.5 \pm 0.4$ & $15.1 \pm 0.6$ & $5.1 \pm 0.2$ & $9.8 \pm 0.3$ & $50.7 \pm 1.2$ & $17.0 \pm 0.5$ & $33.9 \pm 0.8$ \\
\hline$\chi_{r}^{2}$ & 1.3 & 1.2 & 1.5 & 1.1 & 1.0 & 1.2 & 1.5 & 1.3 & 1.6 \\
\hline dof & & 398 & & & 373 & & & 533 & \\
\hline
\end{tabular}

Notes.

${ }^{a}$ Region sizes as fraction of total azimuth are 0.027 (NW), $0.023(\mathrm{NE})$, and $0.032(\mathrm{~W})$

b Column density $\times 10^{21} \mathrm{~cm}^{-2}$.

${ }^{c}$ Model normalization $\times 10^{-3}$.

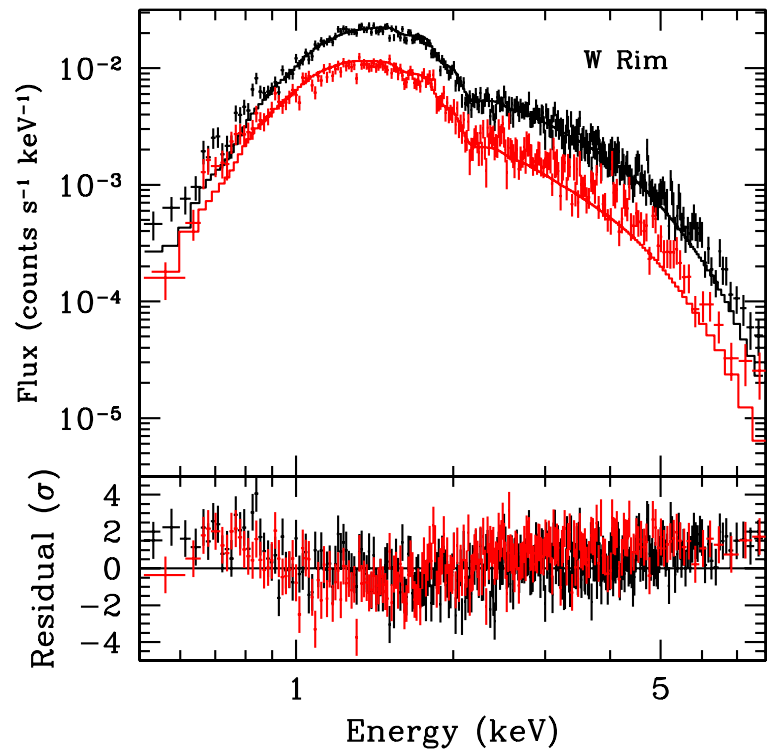

Figure 9. Same as Figure 8, for $\mathrm{W}$ rim of Tycho.

(A color version of this figure is available in the online journal.)

tbabs (Wilms et al. 2000) with the abundance set from Anders \& Grevesse (1989). The normalization of the two emission components were tied together, but allowed to vary along with the absorbing column density. We obtain good fits to the spectra from regions 1 and 2 in Figure 1 using results from Model A (shown as a histogram in each figure), although emission from region 3 contains a distinct excess of soft X-ray emission, discussed in Section 4. Similar features were reported by Hwang et al. (2002). Given the evidence for significant density variations around Tycho (Williams et al. 2013), it is not surprising that some regions contain more shocked ISM material than predicted by our one-dimensional (1D) models which average over the entire SNR. The fit parameters for regions 1 and 2 are summarized in Table 2; region 3 was eliminated from these fits because of clear ejecta contamination (see Section 4). The results from fits to Model B (see Table 2) are similar to that from Model A; the goodness-of-fit is actually slightly better than for Model A, primarily due to a slightly flatter synchrotron component.

Because our model is spherically symmetric, the expected normalization from the spectral fits is simply the fraction of the total azimuth of the SNR covered by our extraction regions. For region 1, we find that the normalization is in excellent agreement with the expected geometric value in the NE region, but is a factor of $2.6(3.1)$ higher in the NW (W) region. This

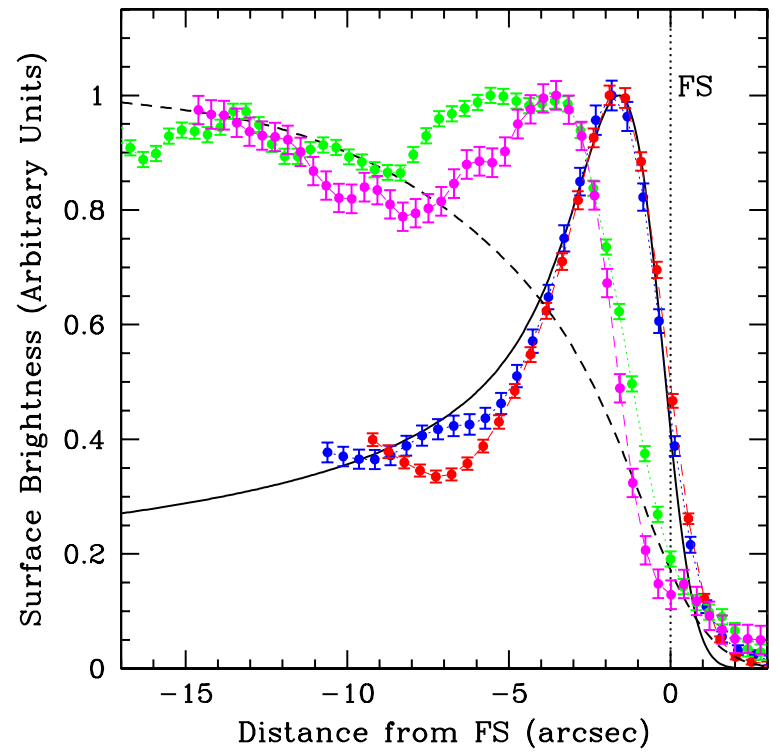

Figure 10. Observed X-ray and radio brightness profiles at the NE/W rims of Tycho, compared with predictions from Model A, described in the text. Blue/ red (green/magenta) points correspond to X-ray (radio) data from the NE/W. Distances are measured from the position of the FS. X-ray and radio data have been renormalized to produce a peak brightness value of 1 , and positions relative to the shock position have been shifted to best align with data with the model profiles.

(A color version of this figure is available in the online journal.)

is consistent with typical variations in the brightness around the rim of Tycho. Moreover, since we have selected brighter filaments in order to obtain good spectra in small regions, it is not surprising that the results are biased to normalizations somewhat higher than expected. More notable is the fact that the normalizations for region 2 falls below that for region 1 in all three regions of the SNR. We find the same behavior for region 3.

The radial brightness profiles for the $\mathrm{NE}$ and $\mathrm{W}$ rims are shown in Figure 10, where blue/red points correspond to $\mathrm{X}$-ray data, and green/magenta points represent radio profiles. The accompanying curves are the predicted brightness profiles from our Model A. The predicted X-ray profile is in reasonable good agreement with the observations near the shock, but declines more slowly than the observed brightness at larger distances behind the shock, consistent with the relatively lower normalizations obtained in spectral fits from these regions. Meanwhile the predicted radio profile, while correctly indicating a slow rise to a plateau-like region well behind the shock, is less 

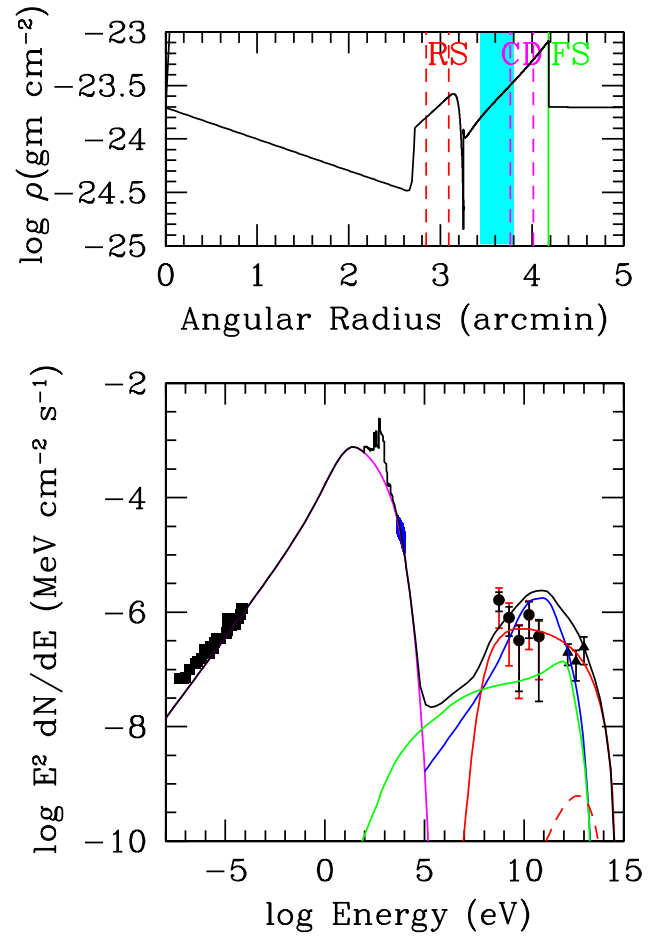

Figure 11. Same as Figure 3, but for Model C.

(A color version of this figure is available in the online journal.)

successful at reproducing the exact profile. This behavior is similar to that found by Cassam-Chenaï et al. (2007). Because the radio-emitting electrons do not suffer significant radiative losses, the projected brightness profile should show a continuous increase from the FS to the CD. The observed climb to an early plateau may be an indication that these electrons are confined to a shell that is considerably thinner than the region between the FS and CD in our model. The physical mechanism associated with such a picture is not obvious, although a reduction in the FS/CD separation from R-T structures described above could be partially responsible. Alternatively, the radial distribution of the magnetic field in the postshock region may deviate from that in our model. Cassam-Chenaï et al. (2007) suggest, for example, that rapid damping of the field at the FS after its rapid initial rise (Pohl et al. 2005) could produce both the sharp rise and the partial falloff of the radio emission, and that turbulent motions from the outermost ejecta could enhance the downstream field, thus resulting in an increase in the radio synchrotron brightness in this region. The corresponding profiles from the NW region are complicated by overlapping shocks that are evident in Figure 1, and are thus not shown in Figure 10.

\subsection{Additional Models}

As noted in Section 1, models of the ejecta emission from Tycho by Badenes et al. (2006) appear to require a somewhat higher density than those derived from X-ray (Cassam-Chenaï et al. 2007; Katsuda et al. 2010) and IR measurements (Williams et al. 2013). Figure 11 presents our best results for a fixed ambient density of $n_{0}=0.85 \mathrm{~cm}^{-3}$ (our Model C), the lowest value reported by Badenes et al. (2006). The CD is located quite far behind the position estimated by Warren et al. (2005), even if the effects of R-T instabilities are considered, although it is important to note that the exponential density profile assumed here differs from that used by Badenes et al. (2006), which was based on actual explosion models with stratified composition.
The radio and $\gamma$-ray components from the higher-density model also provide poor fits to the data. The integrated X-ray spectrum from Model C predicts significant line emission, but the overall contribution of this component to the small regions studied here is small; the X-ray fits are formally worse than for Models A and $\mathrm{B}$, but the differences are not dramatic. Thus, while we view the high density model case to be very unlikely based on both the broadband emission and the dynamical evolution of the shocks, it cannot be ruled out by the X-ray data alone. The parameters for Model $\mathrm{C}$ are summarized in Table 1.

Most recently Chiotellis et al. (2013) have revisited the investigation of the RS spectrum for Tycho for models that include initial evolution in a small wind cavity. They find that the resulting ionization characteristics provide a somewhat better match than that obtained by Badenes et al. (2006) with a modest ambient density of $\sim 0.4 \mathrm{~cm}^{-3}$ and the presence of a wind-blown bubble through which the remnant evolves at an early age. In this model, the ambient density outside the cavity can be somewhat low, as indicated by the X-ray and IR measurements, while the effective density associated with the ionization can be somewhat higher due to the initial expansion through the inner wind region. As this model does not consider DSA, it cannot explain the observed $\gamma$-ray emission. Moreover, with DSA included, the proposed density would result in a FS radius that is smaller than that observed, as discussed in Section 1. We have thus investigated a similar scenario, also considering the effects of efficient DSA on the dynamical evolution. Specifically, we inserted a relic bubble with radius $0.4 \mathrm{pc}$, blown by a stellar wind with $\dot{M}_{w}=3 \times 10^{-6} M_{\odot} \mathrm{yr}^{-1}$ and $v_{w}=10 \mathrm{~km} \mathrm{~s}^{-1}$, and allowed the SNR to evolve through the bubble and into a uniform ambient medium. We find that using parameters similar to those from Model A, along with the inclusion of the wind bubble, yields virtually no change in our results; a slightly lower density is required $\left(n_{0}=0.28 \mathrm{~cm}^{-3}\right)$, and the density profile shows structure from reflected shocks associated with the wind bubble interaction, but the overall dynamics are nearly identical and the broadband spectrum is indistinguishable from that of Model A. This is to be expected, because the total mass contained in the wind component is only $\sim 0.1 M_{\odot}$, while the total sweptup mass in Model A is $\sim 2.5 M_{\odot}$ (see Table 1). Thus, while an early effect on the ionization of the ejecta (which we do not treat here) appears plausible, the impact on the dynamical evolution is negligible. Thus, we conclude that the wind bubble model proposed by Chiotellis et al. (2013) can produce results consistent with current dynamical and spectral measurements of Tycho, though when the effects of CR acceleration are taken into account, the required ambient density is $\lesssim 0.3 \mathrm{~cm}^{-3}$.

\section{DISCUSSION}

Based upon our Model A, we conclude that Tycho's SNR has evolved in a medium with an ambient density $n_{0} \sim 0.3 \mathrm{~cm}^{-3}$ and a magnetic field strength $B \sim 5 \mu \mathrm{G}$. The remnant has undergone efficient acceleration of electrons and ions, with $\sim 16 \%$ of the kinetic energy of the supernova explosion being deposited into relativistic particles with $K_{\text {ep }} \sim 0.003$. The DSA efficiency is $\sim 26 \%$ at the current epoch, the amplified magnetic field in the immediate postshock region has a strength of $\sim 180 \mu \mathrm{G}$, and roughly $11 \%$ of the energy that has gone into particle acceleration has been lost to escaping particles. The remnant distance is $\sim 3.2 \mathrm{kpc}$. This model is consistent with the measured positions and expansion speeds of the FS and RS in Tycho.

As noted in Section 3.1, with our models tuned to produce the observed FS radius at the known age of Tycho, our Model A 


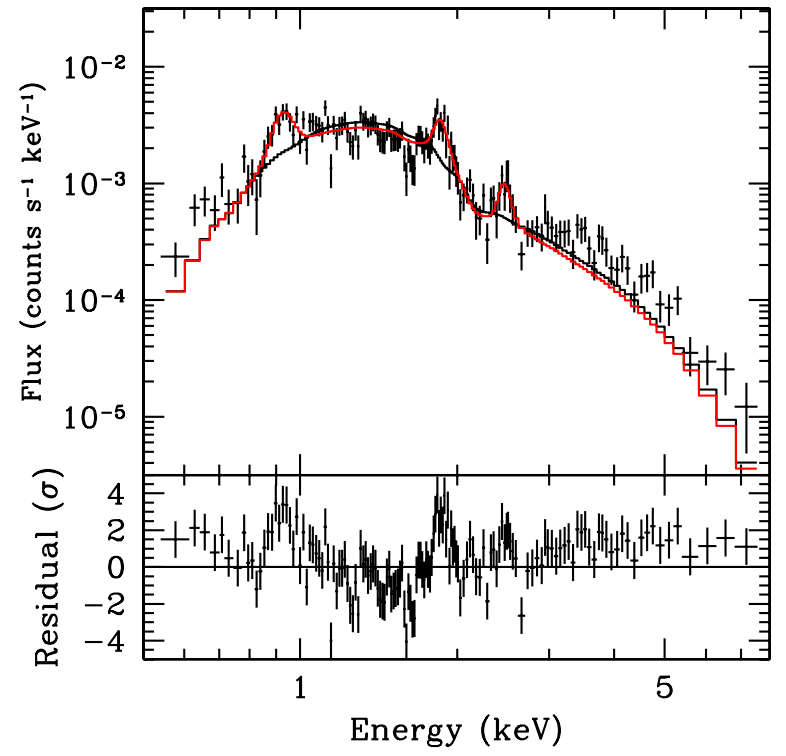

Figure 12. Spectrum from NE region 3 from Tycho (see Figure 1). The black histogram corresponds to the best-fit absorbed CR-hydro-NEI model for the corresponding projected region. Residual emission from $\mathrm{Ne}, \mathrm{Si}$, and $\mathrm{S}$ are evident, indicating the presence of ejecta extended nearly all the way to the FS. The red histogram corresponds to a CR-hydro-NEI model with Gaussians added at the energies expected for $\mathrm{He}$-like emission from $\mathrm{Ne}, \mathrm{Si}$, and $\mathrm{S}$, although we note that significant Ne emission is not expected in spectra of Type Ia SNRs.

(A color version of this figure is available in the online journal.)

reproduces the observed RS radius, but yields a $\mathrm{CD}$ position that falls short of that estimated by Warren et al. (2005). We suggested that R-T filaments have resulted in ejecta material being mixed well beyond the CD, toward the FS. In Figure 12 we show a spectrum from region 3 in the NE section of Tycho (see Figure 1) along with our best-fit CR-hydro-NEI model (black histogram). Significant residuals can be seen at the positions of emission lines from $\mathrm{Ne}(0.92 \mathrm{keV}), \mathrm{Si}(1.85 \mathrm{keV})$, and $\mathrm{S}$ $(2.45 \mathrm{keV})$, which were subsequently fit by Gaussians with energies fixed at the line energies for these elements. Similar results were reported by Hwang et al. (2002) and Cassam-Chenaï et al. (2007), and provide strong evidence for the presence of ejecta mixed nearly all the way to the FS, although the presence of significant amounts of $\mathrm{Ne}$ is quite unexpected given the much lower abundance in nucleosynthesis models for SN Ia. It must be noted that there are numerous Fe-L features in the region around $1 \mathrm{keV}$, which complicates the interpretation of this feature.

The broadband emission from Tycho's SNR has been modeled by a number of researchers (e.g., Morlino \& Caprioli 2012; Atoyan \& Dermer 2012; Berezhko et al. 2013; Zhang et al. 2013) with most concluding that the $\gamma$-ray emission is dominated by pion decay. We reach a similar conclusion, although there are significant differences between our model and previous ones. First, unlike all other models, we simultaneously fit the broadband continuum and X-ray line emission with a single self-consistent model. As has been shown previously (e.g., Ellison et al. 2007, 2010; Patnaude et al. 2009; Lee et al. 2012, 2013; Castro et al. 2012), the X-ray observations strongly constrain the ambient density and can provide a distinction between pion decay and IC emission at $\gamma$-ray energies. Second, we are able to obtain a satisfactory broadband fit without invoking an arbitrary multi-component environment where some fraction of the swept-up mass is in dense clumps as is done in Berezhko et al. (2013). Of course, we do not argue that dense clumps do not exist; the morphology of any SNRs is more complicated than the spherically symmetric model we use. At present, however, observational evidence of any such clumpy environment surrounding Tycho is insufficient to provide constrained parameters for such modeling. From a modeling perspective, there is thus little motivation to add a second component with an additional set of unconstrained parameters when a one-component model produces a satisfactory fit.

It has been argued (Atoyan \& Dermer 2012) that a two-zone model in which electrons accelerated at the FS shock emit in two distinct environments provides a more realistic picture for Tycho's SNR than the many one-zone models that have been considered. In this scenario, a thin outer zone at the FS produces the X-ray synchrotron emission while, inside the FS, electrons accumulate in a region of lower B-field. The IC emission is produced from electrons in both zones, while the synchrotron emission arises primarily from the outer zone. Using several unconstrained parameters to describe the zones, Atoyan \& Dermer (2012) can fit the broadband continuum with the $\gamma$-ray emission dominated by IC but they note that hadronic models are equally viable. As noted above, our evolving, continuous zone, spherically symmetric model also has electrons (and ions) radiating in different environments, shocked at different times, where the parameters are determined from the hydro simulation coupled to the DSA calculation.

The most complete models of Tycho were presented by Morlino \& Caprioli (2012) and Berezhko et al. (2013). Berezhko et al. (2013) criticize Morlino \& Caprioli (2012) for their presumably inconsistent treatment of diffusion. Particle diffusion is indeed critical for DSA; our treatment is described in detail in Lee et al. (2012). Briefly, the CR-hydro-NEI simulation includes a description of magnetic field amplification (MFA) in the shock precursor, so the B-field turbulence is determined as a function of position in the precursor. Our diffusion coefficient is $D(x)=(v p c) /[3 e \delta B(x)]$. The strength of the field variation $\delta B$ from MFA decreases with position in the precursor as the freeescape boundary (FEB) is approached, resulting in an increase in $D$ (i.e., scattering weakens) near the FEB.

Our model also includes non-adiabatic heating in the shock precursor. The MFA produces turbulence, and the dissipation of turbulent energy and heating of the background flow is parameterized. The Alfvén wave speed, which impacts the effective compression ratio for DSA, also varies with position in the precursor. Importantly, there is feedback between the shape and normalization of the accelerated particle spectrum, the MFA and precursor heating, the speed of the Alfvén scattering centers, the normalization of $D$ as a function of position in the precursor, and $p_{\max }$.

We note that $C R$-hydro-NEI also addresses the main concern Berezhko et al. (2013) have concerning the Morlino \& Caprioli (2012) model, namely that the latter authors assume a steep spectrum for the CR protons, in order to match the observed spectrum at lower energies, but then assumed Bohm diffusion for even the highest energy particles in order to obtain emission of $\gamma$-rays beyond $400 \mathrm{MeV}$. This leads to a maximum proton momentum $p_{\max } \sim 500 \mathrm{TeV}$. Berezhko et al. (2013) note that Bohm diffusion is inconsistent with such a steep spectrum, and argue that a two-component spectrum is required. Here, we do not make the same assumption. The spectrum of the accelerated particles, the normalization of the diffusion coefficient, and the resulting maximum momentum are all calculated selfconsistently. As noted above, we obtain $p_{\max } \sim 50 \mathrm{TeV}$ while still reproducing the observed broadband spectrum. In short, we believe the CR-hydro-NEI model addresses all of the criticisms 
Berezhko et al. (2013) make of the Morlino \& Caprioli (2012) model, for which the results of our more detailed modeling are in good agreement.

In the results presented here, we do make the Bohm assumption, i.e., that the diffusion coefficient is equal to the particle gyroradius. However, the gyroradius is determined with the local amplified $\delta B(x)$ which varies with precursor position. At this point, we do not believe that generalizing our fits to include a parameter search with an arbitrary momentum dependence on the gyroradius is warranted.

\section{CONCLUSIONS}

We have carried out a detailed study of the radial structure, evolution, and broadband emission from Tycho's SNR. Using our CR-hydro-NEI code, we have investigated a range of parameters to identify solutions that reproduce the observed dynamical properties of the remnant and reproduce the observed broadband emission. A key goal of our work was to assess the nature of the observed $\gamma$-ray emission. We find that, for our most successful model, this emission is dominated by $\pi^{0}$-decay resulting from the collisions of relativistic protons accelerated at the FS, with a significant contribution at $\mathrm{GeV}$ energies arising from IC scatting of an ambient photon field dominated by the CMB and local IR emission from Tycho itself. The electronto-proton ratio for injected particles is $\sim 0.003$. Roughly $16 \%$ of the SNR kinetic energy has been converted into relativistic particles, and the impact of this on the SNR evolution is a FS/RS radius ratio that is smaller than what would result in a situation without particle acceleration. This model is also consistent with the measured positions and expansion speeds of the FS and RS in Tycho, and predicts protons accelerated to energies as high as $\sim 50 \mathrm{TeV}$. The distance implied by this model is $\sim 3.2 \mathrm{kpc}$.

The projected brightness profiles from our 1D model are in reasonable agreement with those observed in the radio and $\mathrm{X}$-ray bands at discrete regions along the SNR rim. The predicted X-ray spectrum from (projected) regions along the FS are in good agreement with that observed by Chandra, while that from some regions immediately behind the shock show evidence for density variations around Tycho that are expected based on other studies (e.g., Williams et al. 2013).

The parameters we derive to explain the evolutionary state and history for Tycho are in very good agreement with those from other works that present models in which the $\gamma$-ray emission arises predominantly from $\pi^{0}$-decay, particular those presented by Morlino \& Caprioli (2012). Given our more complete treatment of the evolution and emission, we conclude that these results are robust and provide conclusive evidence for efficient acceleration of CR electrons and ions in Tycho's SNR.

The authors acknowledge important discussions with Brian Williams and Carles Badenes concerning this work. P.S. acknowledges support from NASA Contract NAS8-03060. D.C.E. acknowledges support from NASA grant NNX11AE03G. J.P.H. acknowledges support from Chandra grant GO9-0078X and NASA Suzaku grant NNX08AZ86G.

\section{REFERENCES}

Abdo, A. A., Ackermann, M., Ajello, M., et al. 2009, ApJL, 706, L1 Abdo, A. A., Ackermann, M., Ajello, M., et al. 2010, ApJL, 710, L92 Acciari, V. A., Aliu, E., Arlen, T., et al. 2011, ApJL, 730, 20

Ackermann, M., Ajello, M., Allafort, A., et al. 2013, Sci, 339, 807

Anders, E., \& Grevesse, N. 1989, GeCoA, 53, 197

Atoyan, A., \& Dermer, C. D. 2012, ApJL, 749, L26

Auchettl, K., Slane, P., \& Castro, D. 2014, ApJ, in press

Badenes, C., Borkowski, K. J., Hughes, J. P., Hwang, U., \& Bravo, E. 2006, ApJ, 645,1373

Berezhko, E. G., Ksenofontov, L. T., \& Völk, H. J. 2013, ApJ, 763, 14

Blasi, P., Gabici, S., \& Vannoni, G. 2005, MNRAS, 361, 907

Blondin, J. M., \& Ellison, D. C. 2001, ApJ, 560, 244

Bykov, A. M., Ellison, D. C., Osipov, S. M., Pavlov, G. G., \& Uvarov, Y. A. 2011, ApJL, 735, L40

Caprioli, D., Blasi, P., Amato, E., \& Vietri, M. 2009, MNRAS, 395, 895

Cassam-Chenaï, G., Decourchelle, A., Ballet, J., et al. 2004, A\&A, 414, 545

Cassam-Chenaï, G., Hughes, J. P., Ballet, J., \& Decourchelle, A. 2007, ApJ, 665,315

Castro, D., \& Slane, P. 2010, ApJ, 717, 372

Castro, D., Slane, P., Carlton, A., \& Figueroa-Feliciano, E. 2013, ApJ, 774,36

Castro, D., Slane, P., Ellison, D. C., \& Patnaude, D. J. 2012, ApJ, 756, 88

Chiotellis, A., Kosenko, D., Schure, K. M., Vink, J., \& Kaastra, J. S. 2013, MNRAS, 435, 1659

Decourchelle, A., Ellison, D. C., \& Ballet, J. 2000, ApJL, 543, L57

Decourchelle, A., Sauvageot, J. L., Audard, M., et al. 2001, A\&A, 365, L218

Ellison, D. C., Decourchelle, A., \& Ballet, J. 2004, A\&A, 413, 189

Ellison, D. C., Patnaude, D. J., Slane, P., Blasi, P., \& Gabici, S. 2007, ApJ, 661,879

Ellison, D. C., Patnaude, D. J., Slane, P., \& Raymond, J. 2010, ApJ, 712,287

Ellison, D. C., Slane, P., Patnaude, D. J., \& Bykov, A. M. 2012, ApJ, 744,39

Eriksen, K. A., Hughes, J. P., Badenes, C., et al. 2011, ApJL, 728, L28

Giordano, F., Naumann-Godo, M., Ballet, J., et al. 2012, ApJL, 744, L2

Giuliani, A., Cardillo, M., Tavani, M., et al. 2011, ApJL, 742, L30

Hayato, A., Yamaguchi, H., Tamagawa, T., et al. 2010, ApJ, 725, 894

Hughes, J. P., Rakowski, C. E., \& Decourchelle, A. 2000, ApJL, 543, L61

Hwang, U., Decourchelle, A., Holt, S. S., \& Petre, R. 2002, ApJ, 581, 1101

Hwang, U., \& Gotthelf, E. V. 1997, ApJ, 475, 665

Inoue, T., Yamazaki, R., Inutsuka, S.-i., \& Fukui, Y. 2012, ApJ, 744, 71

Ishihara, D., Kaneda, H., Furuzawa, A., et al. 2010, A\&A, 521, L61

Kamae, T., Karlsson, N., Mizuno, T., Abe, T., \& Koi, T. 2006, ApJ, 647, 692

Katsuda, S., Petre, R., Hughes, J. P., et al. 2010, ApJ, 709, 1387

Krause, O., Tanaka, M., Usuda, T., et al. 2008, Natur, 456, 617

Lee, S.-H., Ellison, D. C., \& Nagataki, S. 2012, ApJ, 750, 156

Lee, S.-H., Slane, P. O., Ellison, D. C., Nagataki, S., \& Patnaude, D. J. 2013, ApJ, 767,20

Morlino, G., \& Caprioli, D. 2012, A\&A, 538, A81

Patnaude, D. J., Ellison, D. C., \& Slane, P. 2009, ApJ, 696, 1956

Pohl, M., Yan, H., \& Lazarian, A. 2005, ApJL, 626, L101

Reynolds, S. P., \& Ellison, D. C. 1992, ApJL, 399, L75

Ruiz-Lapuente, P. 2004, ApJ, 612, 357

Tian, W. W., \& Leahy, D. A. 2011, ApJL, 729, L15

Truelove, J. K., \& McKee, C. F. 1999, ApJS, 120, 299

Völk, H. J., Berezhko, E. G., \& Ksenofontov, L. T. 2008, A\&A, 483, 529

Wang, C., \& Chevalier, R. A. 2001, ApJ, 549, 1119

Warren, D. C., \& Blondin, J. M. 2013, MNRAS, 429, 3099

Warren, J. S., Hughes, J. P., Badenes, C., et al. 2005, ApJ, 634, 376

Williams, B. J., Borkowski, K. J., Ghavamian, P., et al. 2013, ApJ, 770, 129

Wilms, J., Allen, A., \& McCray, R. 2000, ApJ, 542, 914

Yamaguchi, H., Eriksen, K. A., Badenes, C., et al. 2014, ApJ, 780, 136

Zhang, X., Chen, Y., Li, H., \& Zhou, X. 2013, MNRAS, 429, L25 